Paper

\title{
Decreased Fronto-Parietal and Increased Default Mode Network Activation is Associated with Subtle Cognitive Deficits in Elderly Controls
}

\author{
Davide Zanchi ${ }^{a}$ Marie-Louise Montandon ${ }^{\mathrm{b}}$ Indrit Sinanaj ${ }^{\mathrm{b}} \quad$ Cristelle Rodriguez $^{\mathrm{b}}$ \\ Antoinette Depoorter ${ }^{c}$ Francois R. Herrmann ${ }^{d}$ Stefan Borgwardt ${ }^{a}$ \\ Panteleimon Giannakopoulos ${ }^{b}$ Sven Halleref,g \\ aDepartment of Psychiatry (UPK), University of Basel, Basel, bepartment of Psychiatry, Faculty of \\ Medicine of the University of Geneva and Medical Direction, University of Geneva Hospitals, 'Division \\ of Neuropediatrics \& Developmental Medicine, University Children's Hospital, Basel, dDivision of \\ Geriatrics, Department of internal medicine, rehabilitation and geriatrics, Geneva University Hospitals

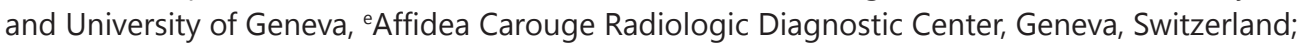 \\ fDepartment of Surgical Sciences, Radiology, Uppsala University, Uppsala, Sweden; 9Department of \\ Neuroradiology, University Hospital Freiburg, Germany
}

\section{Key Words}

Working memory • Alzheimer • fsI FEAT • MCI • Cognitive impairment • Deteriorating controls $\cdot \mathrm{n}$-back

\begin{abstract}
Background: Cognitive functions progressively deteriorate during aging and neurodegenerative diseases. The present study aims at investigating differences in working memory performance as well as functional brain changes during the earliest stages of cognitive decline in health elderly individuals. Methods: 62 elderly individuals (41 females), including 41 controls (35 females) and 21 middle cognitive impairment subjects ( 6 females), underwent neuropsychological assessment at baseline and an fMRI examination in a N-back paradigm contrasting 2-back vs. 0-back condition. Upon a 18 months follow-up, we identified stable controls $(\mathrm{sCON})$ with preserved cognition and deteriorating controls ( $\mathrm{dCON}$ ) with -1SD decrease of performances in at least two neuropsychological tests. Data analyses included accuracy and reaction time (RT) for the 2-back condition and general linear model (GLM) for the fMRI sequence. Results: At the behavioral level, sCON and dCON performed better than MCI in terms of accuracy and reaction time. At the brain level, functional differences in regions of the fronto-parietal network (FPN) and of the Default Mode Network (DFM) were observed. Significantly lower neural activations in the bilateral inferior and middle frontal gyri were found in $\mathrm{MCI}$ versus both dCON / sCON and for dCON versus sCON. Significantly increased activations in the anterior cingulate cortex and posterior cingulate cortex and bilateral insula were found


Zanchi et al:: Working Memory Performance and Changes in Neural Activation in Cognitively Preserved Elders

in $\mathrm{MCI}$ versus both dCON / sCON and in dCON versus sCON. Conclusion: The present study suggests that brain functional changes in FPN and DMN anticipate differences in cognitive performance in healthy elderly individuals with subsequent subtle cognitive decline.

\section{Introduction}

Cognitive functions progressively deteriorate during aging and in particular during neurodegenerative diseases. Accordingly to a milestone work by Jack and colleagues [1] this cognitive deterioration is the later consequence of the early appearance at the brain level of amyloid plaques, followed by grey matter atrophy of the limbic system and finally by memory loss. Cognitive functions include several domains, including attention, cognitive control and working memory [2], the latter being one of the most affected domain during aging [3].

Working memory is defined as 'a brain system that provides temporary storage and manipulation of the information necessary for complex cognitive tasks' [4]. Efficient working memory (WM) performance relies on the connectivity of at least two large-scale networks operating in concert: the default mode network (DMN) and the fronto-parietal network (FPN). The DMN involves bilateral and symmetrical cortical areas in the medial and lateral parietal, medial prefrontal, and medial and lateral temporal cortices [5]. It is usually more active during rest and may change its connectivity during WM tasks [6-8]. The FPN comprises fronto-parietal regions such as the superior, middle and inferior frontal gyri in addition to the inferior parietal lobules It is typically activated during executive functions and its integrity is critical for the successful performance of WM tasks $[9,10]$.

Several neuroimaging studies revealed altered activation of FPN and DMN both in mild cognitive impairment (MCI) and Alzheimer disease (AD) that affects performances in highly demanding WM tasks $[11,12]$. In particular, during the widely disseminated N-back task, higher activation was found in the right superior frontal gyrus, middle frontal gyrus and anterior cingulate cortex in mild cognitive impairment (MCI) and AD compared to controls [12]. Compared to MCI cases, AD patients showed reduced activations in the right parahippocampal, left inferior frontal and cingulate gyrus $[12,13]$. To date, the focus of interest is moving from MCI cases that display a substantial brain compromise to pre-MCI cases with very subtle cognitive deficits $[14,15]$. In the context of a large community-based study of brain aging in Geneva, we identified a population of deteriorating controls (dCON), i.e. individuals with intact cognitive tests at baseline who display very subtle cognitive decline $(-0.5 \mathrm{SD}$ in at least two cognitive tasks) compatible to the pre-MCI state at 18 month followup [16].

In order to explore WM-related brain network tasks in early phases of cognitive decline that precede $\mathrm{MCI}$, we examined the patterns of fMRI activation during the N-back task in dCON compared to healthy controls with stable cognitive performances at follow-up. Our data document significant changes in FPN and DMN activation at baseline in cases that subsequently develop very subtle cognitive deficits.

\section{Materials and Methods}

\section{Participants}

After formal approval by the local Ethics Committee, 62 elderly individuals were included in the study after providing informed written consent prior to inclusion. These community-based cases were recruited via advertisements in local newspapers and media. All participants had normal or corrected-to-normal visual acuity. Past hearing problems were identified as a part of the medical interview (including both subjects and their proxies). All cases with such problems were a priori excluded. Audition was tested by standard audiologic tests including self-report and speech in noise perception in all cases during clinical routine 
medical examination [17]. Cases with self-report of hearing loss and altered speech in noise perception were addressed in specialized consultation and were not considered for further investigations. The education level was defined according to the Swiss scholar system, where level 1 = less than 9 years (primary school), level 2 = between 9 and 12 years (high school) and level 3 = more than 12 years (university).

The neuropsychological batteries at baseline and follow-up were identical. At baseline, all individuals were evaluated with an extensive neuropsychological battery, including the Mini-Mental State Examination (MMSE) [18], the Hospital Anxiety and Depression Scale [19] and the Lawton Instrumental Activities of Daily Living [20]. Cognitive assessment included (a) attention (Digit-Symbol-Coding, Trail Making Test A [21], (b) working memory (verbal: Digit Span Forward [22], visuo-spatial: Visual Memory Span (Corsi) [23], (c) episodic memory (verbal: RI-48 Cued Recall Test [24] or RL/RI-16 Free and Cued Recall Test, visual: Shapes Test [4], (d) executive functions (Trail Making Test B[21]), Wisconsin Card Sorting Test and Phonemic Verbal Fluency Test), (e) language (Boston Naming[25]), (f) visual gnosis (Ghent Overlapping Figures), (g) praxis: ideomotor [26], reflexive [27], and constructional [28], Figures copy [28]. All individuals were also evaluated with the Clinical Dementia Rating scale[29]. Those who met dementia DSM-IV diagnostic criteria on the basis of the neuropsychological and clinical assessments were excluded.

In agreement with the criteria of Petersen et al. [30], participants with a CDR score of 0.5 but no dementia and a score more than 1.5 standard deviations below the age-appropriate mean in any of the previously mentioned tests were classified as MCI. Participants with neither dementia nor MCI were classified as cognitively healthy older controls and underwent full neuropsychological assessment at follow-up, on average 18 months later. Those whose cognitive scores remained stable and those whose performance at follow-up was at least 0.5 SD lower compared to the baseline evaluation in at least two cognitive tests, were classified as stable $(\mathrm{sCON})$ and deteriorating $(\mathrm{dCON})$, respectively.

After neuropsychological evaluations, the final sample consisted of 22 sCON (mean age $68.7 \pm 2.0,20$ women, MMSE at baseline $28.6 \pm 1.7$ ), 19 dCON (mean age $68.8 \pm 4.1,15$ women, MMSE at baseline $28.3 \pm 1.4$ ) and $21 \mathrm{MCI}$ (mean age $73.5 \pm 5.8,6$ females, MMSE at baseline $27.0 \pm 1.7$ ).

\section{Demographic and Neuropsychological assessment}

Demographic differences in the age of the participants were found between MCI and sCON $(\mathrm{P}<.01)$ and between $\mathrm{MCI}$ and dCON $(\mathrm{P}<.01)$. Gender differences were found between MCI and sCON $(\mathrm{P}<.01)$ and between MCI and dCON $(\mathrm{P}<.01)$. No differences in education levels were found between the three groups. The demographic data of the three groups at baseline

Table 1. Demographics. Mean (standard deviations) of the main demographic characteristics. Analysis of variance (ANOVA) using Tuckey post-hoc multiple comparison corrections was performed

\begin{tabular}{|c|c|c|c|c|c|c|c|c|c|c|c|c|c|c|}
\hline Group & \multicolumn{3}{|c|}{ sCON } & \multicolumn{3}{|c|}{$\mathrm{dCON}$} & \multicolumn{3}{|c|}{$\mathrm{MCI}$} & \multicolumn{3}{|c|}{ Total } & $\begin{array}{c}\begin{array}{c}\text { Group } \\
\text { effect }\end{array} \\
p\end{array}$ & $\begin{array}{c}\begin{array}{c}\text { sCon vs } \\
\mathrm{dCON}\end{array} \\
\mathrm{p}\end{array}$ \\
\hline & $\mathrm{N}$ & & SD & $\mathrm{N}$ & & SD & $\mathrm{N}$ & & SD & $\mathrm{N}$ & & SD & & \\
\hline & Mean & & $\%$ & Mean & & $\%$ & Mean & & $\%$ & Mean & & $\%$ & & \\
\hline $\mathrm{N}$ & 22 & & & 19 & & & 21 & & & 62 & & & & \\
\hline Female sex & 20 & & 90.9 & 15 & & 78.9 & 6 & & 28.6 & 41 & & 66.1 & $<0.0001$ & 0.685 \\
\hline $\begin{array}{l}\text { Education } \\
\text { (year) }\end{array}$ & & & & & & & & & & & & & 0.9123 & 0.699 \\
\hline$<9$ & 1 & & 4.5 & 1 & & 5.3 & 3 & & 14.3 & 5 & & 8.1 & & \\
\hline $9-12$ & 11 & & 50.0 & 12 & & 63.2 & 9 & & 42.9 & 32 & & 51.6 & & \\
\hline$>12$ & 9 & & 40.9 & 6 & & 31.6 & 9 & & 42.9 & 24 & & 38.7 & & \\
\hline Age at MRI & 68.7 & \pm & 2.0 & 68.8 & \pm & 4.1 & 73.5 & \pm & 5.8 & 70.4 & \pm & 4.7 & 0.0005 & 0.9498 \\
\hline
\end{tabular}


Zanchi et al:: Working Memory Performance and Changes in Neural Activation in Cognitively Preserved Elders

Table 2. Baseline characteristics by group (mean \pm SD). Stable ( $\mathrm{sCON}$ ), Deteriorating (dCON), Mild cognitive impairment (MCI)

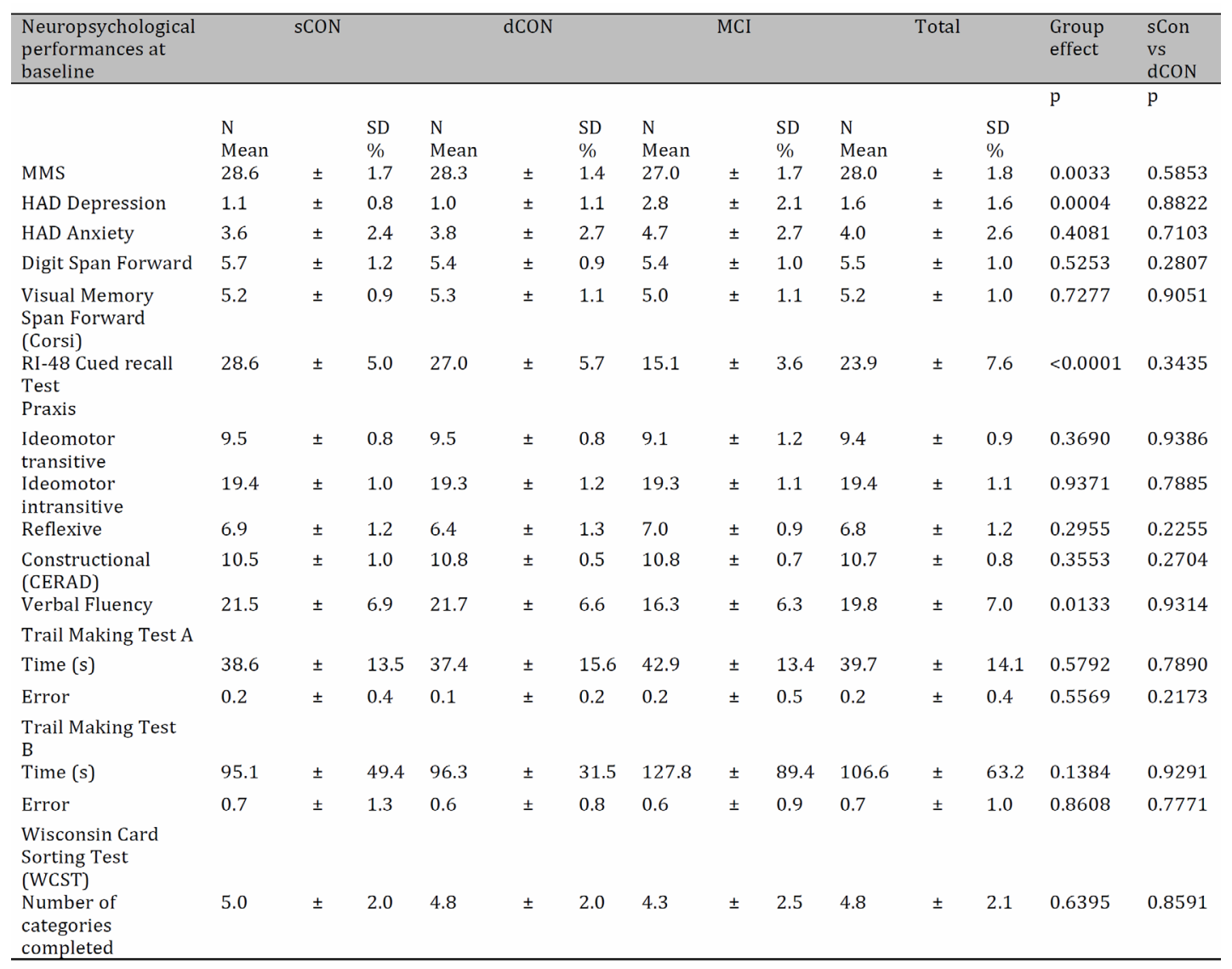

Table 3. Neuropsychological performances at 18 month follow-up by group (mean \pm SD). Stable (sCON), Deteriorating (dCON)

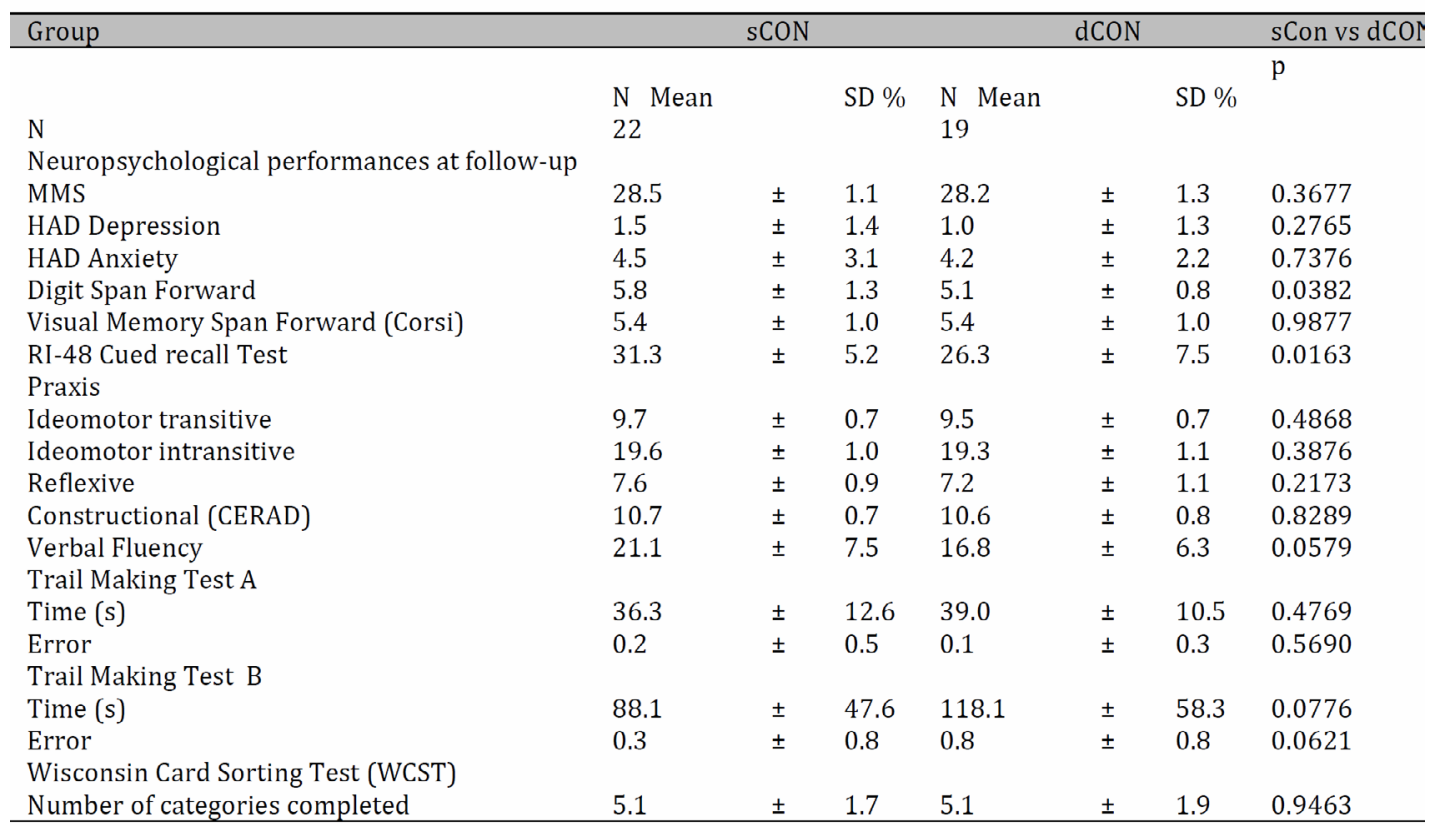


Zanchi et al.: Working Memory Performance and Changes in Neural Activation in Cognitively Preserved Elders

are summarized in Table 1. Neuropsychological performances did not differ between sCON and dCON groups at baseline (Tabel 2). The neuropsychological differences between sCON and dCON at follow-up are summarized in Table 3.

\section{WM task}

All participants performed an $n$-back task, a well-established WM task in fMRI as described previously $[31,32]$. Briefly, a sequence of letters was presented visually on an MR compatible canvas in the MRI scanner. In the active 2-back condition (high WM demand), targets are letters that are identical to the letter presented two items ago (e.g. "a $\mathrm{f} \mathrm{h} \mathrm{f}$ "). In the control condition 0-back (visual processing with minimal WM requirements), the target is a pre-defined letter (e.g. " $\mathrm{x}$ "). Both conditions are contrasted to evaluate the effect of WM demand in 2-back versus the control condition 0-back. Participants were familiarized with the task demands prior to MRI using a training session. The fMRI protocol consisted of four runs of approximately 8-min duration each. Stimuli were pseudorandom sequences of consonants (randomly varying in case), presented in the center of the visual field (500-ms duration, 2500-ms stimulus onset asynchrony). Each run included 10 pseudo-randomized blocks of $35 \mathrm{~s}$ each (five per type of task) with interleaving rest conditions of $15 \mathrm{~s}$ to allow the hemodynamic response to recover from the previous block. Blocks started with a cue screen announcing the type of judgment to perform in the upcoming trials e.g. "0-back" or "2-back". Participants were instructed to emphasize equally accuracy or speed in task completion.

\section{MR imaging}

We used the same scanning protocol as described previously [31, 32]. Functional imaging echo-planar imaging (EPI) time series were acquired on a clinical routine whole body 3.0T MR scanner (TRIO, Siemens medical systems, Erlangen, Germany) with fundamental parameters: echo spacing $=0.86 \mathrm{~ms}$, bandwidth $=1280 \mathrm{~Hz} / \mathrm{px}, \mathrm{TE}=61 \mathrm{~ms}, \mathrm{TR}=2.56 \mathrm{~s}, 122$ ms per slice, flip angle $=90^{\circ}$. After functional scanning, high-resolution anatomical 3DT1 data were acquired ( $1 \mathrm{~mm}$ isotropic T1w MPRAGE, Matrix 256 x 256, 176 slices), which were used for co-registration and spatial normalization.

\section{Statistical analysis}

Statistical analyses were performed using GraphPad Prism (Version 6, GraphPad Software, San Diego, USA), R (Version 0.99.896, The R Project for Statistical Computing) FMRIB Software Library (FSL) (Version 5.0.6, FMRIB, Oxford, UK).

\section{Behavioral data}

The response accuracy (\% of correct answers) and the reaction time for the 2-back and 0-back conditions were analyzed using a two-way ANOVA with Sidak corrections for multiple comparisons.

\section{GLM analysis of task-related activation}

Task-related GLM data processing was carried out using FEAT (FMRI Expert Analysis Tool) version 5.98, part of FSL (FMRIB's Software Library, http://www.fmrib.ox.ac.uk/fsl). Data processing was made according to two levels. At the first level, the contrast of 2-back versus 0-back (and the inverse comparison) was assessed separately for each run of each participant in each group of subjects. At the second level, the contrasts of the diagnostic groups were assessed (sCON versus dCON, sCON versus MCI and dCON versus MCI and the inverse comparisons). Higher-level analysis was carried out using a mixed effect model, by forcing the random effects variance to zero in FLAME (FMRIB's Local Analysis of Mixed Effects). Z statistic images were thresholded using clusters determined by $\mathrm{Z}>2.3$ and a corrected cluster significance threshold of $p=0.05$ was considered as significant. Thresholdfree cluster enhancement (TFCE) multiple comparison correction was used. 


\section{SIGUNAis}

Neurosignals 2017;25:127-138

\begin{tabular}{l|l}
\hline DOI: 10.1159/000486152 & (C) 2017 The Author(s). Published by S. Karger AG, Basel
\end{tabular}

Published online: January 15, 2017

www.karger.com/nsg

Zanchi et al:: Working Memory Performance and Changes in Neural Activation in Cognitively Preserved Elders

\section{Results}

\section{Behavior}

As expected, a main task effect was found $(\mathrm{F}=12.42, \mathrm{p}<.001)$ for all groups and between 0 -back and 2-back conditions sCON ( $<<.001), \mathrm{dCON}(\mathrm{p}<.001)$ and MCI groups $(\mathrm{p}<.001)$. A main group effect was also documented for accuracy $(\mathrm{F}=61.44, \mathrm{p}<.001)$, with significant differences between sCON and MCI $(\mathrm{p}<.001)$ and $\mathrm{dCON}$ and MCI groups $(\mathrm{p}<.001)$ both in 0 -back and for 2-back conditions (Fig. 1A). No group-task interaction was found.

In respect to reaction-time, results of the two-ways ANOVA showed a significant interaction effect between task and group $(\mathrm{F}=3.47)$. In particular, a main task effect was present for reaction time $(\mathrm{F}=3.077, \mathrm{p}<.001)$ and post-hoc analyses revealed increased reaction time in 2-back compared to 0 -back task only in MCI cases $(\mathrm{p}<.001)$. A main group effect for reaction time was also found $(\mathrm{F}=18.82, \mathrm{p}<.001)$, showing differences between sCON and MCI $(\mathrm{p}<.001)$ and $\mathrm{dCON}$ and MCI groups $(\mathrm{p}<.001)$ only within the 2-back condition (Fig. 1B).

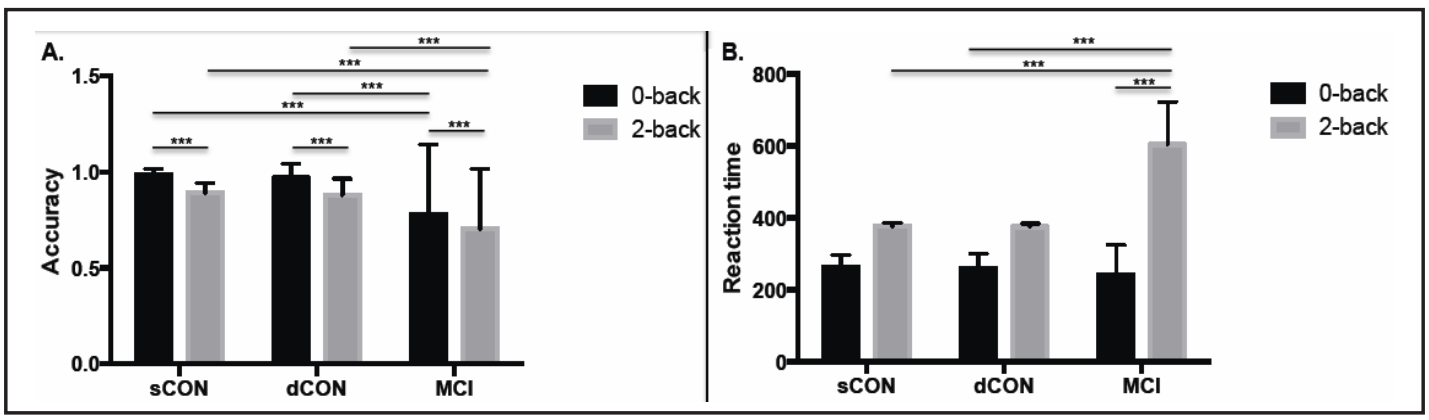

Figure 1. Comparison of behavioral results between the three diagnostic groups according to the N-back tasks.

Fig. 2. Working memory functional changes in the fronto-parietal network. After multiple comparison correction the sCON group showed significantly higher fronto-parietal activation compared to both dCON and MCI. These differences were more pronounced notably in the bilateral superior and middle frontal gyri (Figure 2A, 2B). dCON compared to MCI also showed increased activation notably in the same areas of the FPN (Fig. 2C).

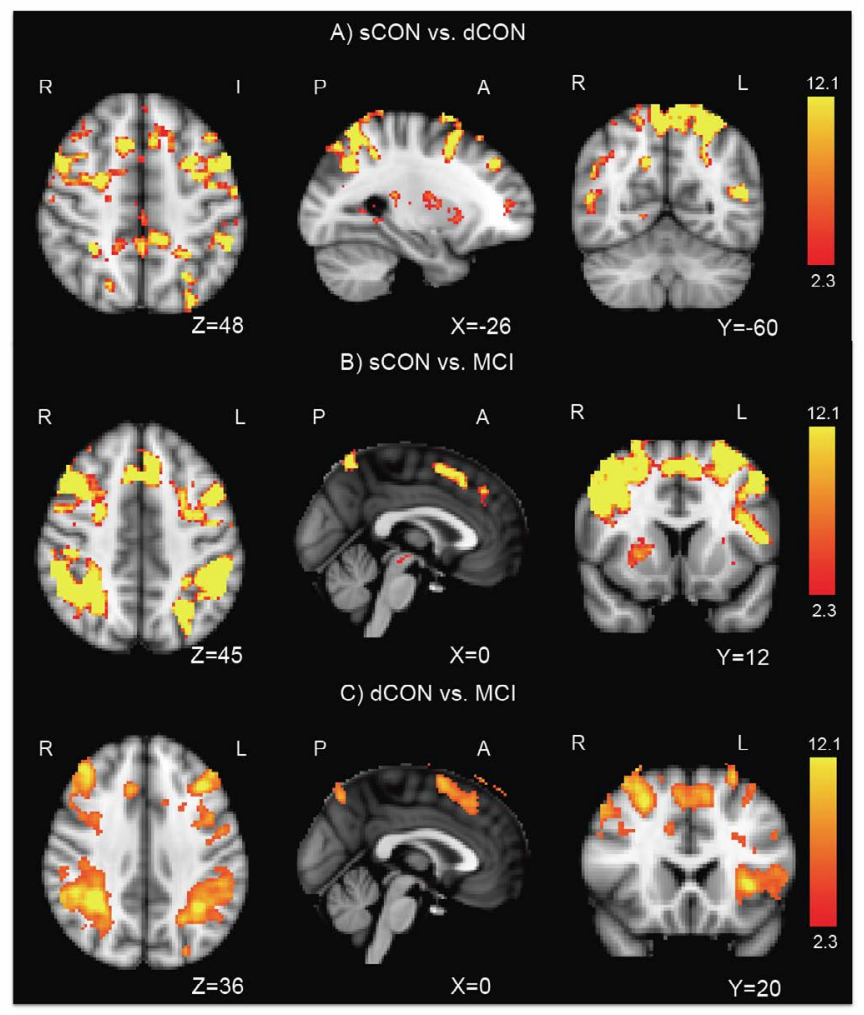


Zanchi et al.: Working Memory Performance and Changes in Neural Activation in

\section{Functional MRI}

After multiple comparison correction the sCON group showed significantly higher fronto-parietal activation compared to both dCON and MCI groups. These differences were more pronounced in the bilateral superior and middle frontal gyri (Fig. 2A, 2B, Table 4, Table 5). Compared to MCI, dCON also showed increased activation of the FPN (Fig. 2C, Table 6).

The contrast 'sCON versus dCON' showed an increased activation of the WM network in sCON mainly in the left fronto-parietal cortex (Fig. 3A, Table 7). The contrast 'MCI vs. $\mathrm{dCON}$ ' and 'MCI vs. sCON' revealed increased activation of the DMN regions including the anterior and posterior cingulate gyrus in addition to the left parahippocampal gyrus (Fig. 3B, 3C Table 8, Table 9).

Table 4. N-back GLM results: sCON vs. dCON

\begin{tabular}{|c|c|c|c|c|c|c|c|c|c|}
\hline Cluster Index & Voxels & $\mathrm{P}$ & $-\log 10(P)$ & Z-MAX & Z-MAX X (mm) & Z-MAXY (mm) & Z-MAX Z (mm) & Side & Regions \\
\hline 1 & 24481 & $9.10 \mathrm{E}-41$ & 40 & 10 & -48 & 6 & 48 & $\mathrm{~L}$ & Superior Frontal Gyrus \\
\hline 2 & $460 \quad 0.0481$ & 1.32 & & 7.16 & -52 & -42 & 48 & $\mathrm{~L}$ & Sensory Cortex \\
\hline
\end{tabular}

Table 5. N-back GLM results: sCON vs. MCI

\begin{tabular}{llllllllll}
\hline Cluster Index & Voxels & P & -log10(P) & Z-MAX & Z-MAXX $(\mathrm{mm})$ & Z-MAXY $(\mathrm{mm})$ & Z-MAXZ $(\mathrm{mm})$ & Side & Regions \\
\hline 1 & 25544 & $5.77 \mathrm{E}-42$ & 41.2 & 12.1 & 40 & 4 & 62 & $\mathrm{R}$ & Superior Frontal Gyrus \\
2 & 891 & 0.00115 & 2.94 & 5.29 & -10 & -10 & 8 & B & Thalamus \\
3 & 816 & 0.0021 & 2.68 & 6.37 & -48 & -60 & -4 & L & Middle Temporal Gyrus \\
\hline
\end{tabular}

Table 6. N-back GLM results: dCON vs. MCI

\begin{tabular}{llllllllll}
\hline Cluster Index & Voxels & $\mathrm{P}$ & $-\log 10(\mathrm{P})$ & Z-MAX & Z-MAXX $(\mathrm{mm})$ & Z-MAXY $(\mathrm{mm})$ & Z-MAX Z (mm) & Side & Regions \\
\hline 1 & 8648 & $6.47 \mathrm{E}-20$ & 19.2 & 11.9 & -44 & -50 & 56 & L & Sensory Cortex \\
2 & 4847 & $3.02 \mathrm{E}-13$ & 12.5 & 9.41 & 42 & 40 & 34 & R & Superior Frontal Gyrus \\
3 & 1997 & $6.56 \mathrm{E}-07$ & 6.18 & 10.7 & -36 & 28 & 34 & L & Superior Frontal Gyrus \\
4 & 564 & 0.0182 & 1.74 & 6.65 & -40 & 4 & 34 & L & Pre-central Gyrus \\
\hline
\end{tabular}

Table 7. N-back GLM results: dCON vs. sCON

\begin{tabular}{llllllllll}
\hline Cluster Index & Voxels & $\mathrm{P}$ & -log10(P) & Z-MAX & Z-MAXX $(\mathrm{mm})$ & Z-MAXY $(\mathrm{mm})$ & Z-MAX Z (mm) & Side & Regions \\
\hline 1 & 772 & 0.00301 & 2.52 & 8.93 & -34 & -50 & 38 & L & Sensory Cortex \\
2 & 724 & 0.00449 & 2.35 & 9.09 & -38 & 30 & 30 & L & Superior Frontal Gyrus \\
3 & 627 & 0.0104 & 1.98 & 6 & -2 & 8 & 64 & B & Pre-central Gyrus \\
\hline
\end{tabular}

Table 8. N-back GLM results: MCI vs. dCON

\begin{tabular}{llllllllll}
\hline Cluster Index & Voxels & $\mathrm{P}$ & $-\log 10(\mathrm{P})$ & Z-MAX & Z-MAXX $(\mathrm{mm})$ & Z-MAXY (mm) & Z-MAXZ(mm) & Side & Regions \\
\hline 1 & 21329 & $4.15 \mathrm{E}-37$ & 36.4 & 8.67 & -2 & -50 & 22 & B & Posterior Cingulate Cortex \\
\hline
\end{tabular}

Table 9. N-back GLM results: MCI vs. sCON

\begin{tabular}{llllllllll}
\hline Cluster Index & Voxels & P & -log10(P) & Z-MAX & Z-MAXX $(\mathrm{mm})$ & Z-MAXY $(\mathrm{mm})$ & Z-MAXZ $(\mathrm{mm})$ & Side & Regions \\
\hline 1 & 4231 & $5.09 \mathrm{E}-12$ & 11.3 & 7.32 & 2 & -46 & 32 & B & Posterior Cingulate Cortex \\
2 & 2005 & $5.96 \mathrm{E}-07$ & 6.22 & 5.06 & -34 & -20 & 18 & L & Frontal Pole \\
3 & 1383 & $3.18 \mathrm{E}-05$ & 4.5 & 5.81 & -24 & 56 & 34 & B & Pre-central Gyrus \\
\hline
\end{tabular}




\section{SIGENAis}

Fig. 3. Working memory functional changes in the default mode network. After multiple comparison correction, the contrast 'dCON versus sCON' showed a increased activation of the WM network in particular in the left fronto-parietal cortex (Fig. 3A). The contrasts 'MCI vs. dCON' and 'MCI vs. sCON' revealed increase activation of the DMN regions as notably the anterior and posterior cingulate gyrus in addition to the left parahippocampal gyrus (Fig. 3B, 3C).

\begin{tabular}{|c|c|}
\hline \multicolumn{2}{|c|}{ Neurosignals 2017;25:127-138 } \\
\hline $\begin{array}{l}\text { DOI: } 10.1159 / 000486152 \\
\text { Published online: January 15, } 2017\end{array}$ & $\begin{array}{l}\text { (c) } 2017 \text { The Author(s). Published by S. Karger AG, Basel } \\
\text { www.karger.com/nsg }\end{array}$ \\
\hline
\end{tabular}

Zanchi et al.: Working Memory Performance and Changes in Neural Activation in Cognitively Preserved Elders

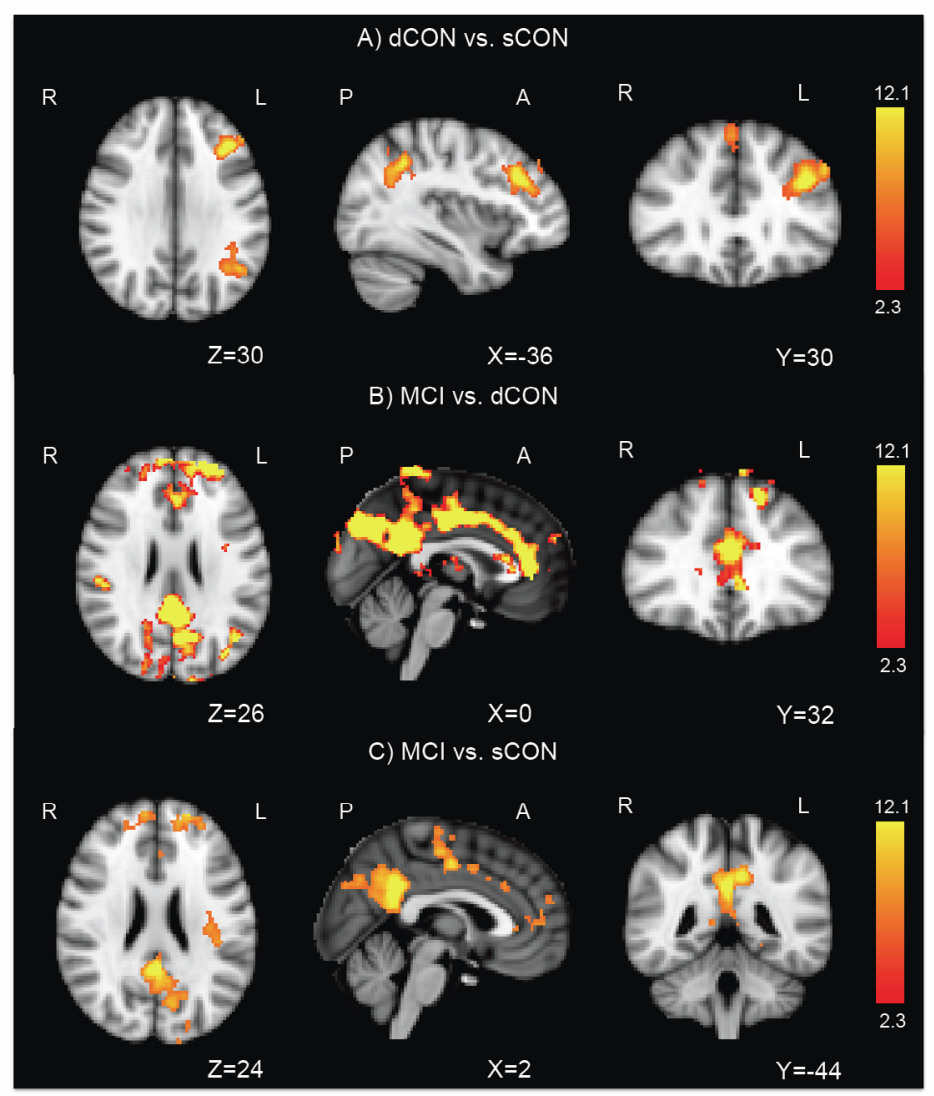

\section{Discussion}

To our knowledge, this is the first study that explores the fMRI patterns of brain activation in n-back task among cognitively unstable individuals prior to the MCI state. Overall, our data reveal two opposite patterns of activation/deactivation in FPN and DMN during the $\mathrm{N}$-back WM task in the initial phases of cognitive decline. Overall, there is a progressive and significant decrement of FPN activation from sCON to dCON and MCI that was mainly observed in the bilateral inferior and middle frontal gyri. In contrast, a significant and gradual DMN increased activation in the ACC and PCC and bilateral insula was documented starting in dCON and becoming more marked in MCI cases. At the behavioral level, sCON and dCON displayed similar performances, significantly better than $\mathrm{MCI}$, both in terms of accuracy and reaction times.

At the behavioral level, the present data show the presence of differences in cognitive performance during the N-back task both for accuracy and reaction time between MCI, sCON and dCON. Significantly worse accuracy was recorded for MCI compared to the other groups for the 2-back task. Moreover, MCI were slower than controls in responding to the 2 back condition, confirming previous results indicating higher efforts for MCI compared to controls during the 2 back condition. These results confirm previous studies suggesting decreased WM performance in MCI cases compared to controls in higher WM load [12, 33, 34]. Importantly and despite the presence of subtle cognitive deficits upon 18-month followup, there were no significant differences in N-back performance at baseline between sCON and dCON.

The main findings of the present study concern the differential patterns of brain activation in FPN and DMN between MCI, dCON and sCON cases during the n-back task. A 
gradual and significant decrease of FPN activation was found in $\mathrm{dCON}$ and $\mathrm{MCI}$ as compared to sCON in the bilateral inferior and middle frontal gyri. In particular, the dCON group exhibited intermediate values of brain activation in FPN, significantly different from both sCON and MCI cases. An inverse scenario was seen in DMN with significantly higher activations in the ACC and PCC and bilateral insula observed in cases with subtle (dCON) and more pronounced (MCI) cognitive decline. Here again, the dCON group can be easily differentiated from MCI cases.

Previous studies showed that decreased activation of FPN is associated with worst WM performances across the lifespan $[35,36]$. Recent studies using near-infrared spectroscopy also indicated decreased frontal activation at high WM load in MCI cases and suggested an association between frontal hypoactivation and WM difficulties[13]. In the same line, an altered frontal lateralization that impact on verbal fluency performances in $\mathrm{MCI}$ cases was recently reported [37]. Moreover, MCI cases exhibit increased difficulties in modulating FPN activation that usually decreases after cognitive training to guarantee processing efficiency in healthy controls [38]. Recent contributions on the role of DMN activity in n-back task pointed to the decrease of its activation and functional connectivity with increased WM load in healthy controls $[39,40]$. Interestingly, the increased activation of DMN in PCC and medial prefrontal cortex is not associated with worst WM performances at least in non-demented cases [41]. In agreement with these findings, our data indicate that decreased FPN and increased DMN activations are present in MCI cases that display decreased accuracy and higher reaction times in their 2-back responses. Most importantly, they suggest that these characteristics of brain activation facing increased WM load is already present at the preMCI state despite preserved n-back performances.

\section{Limitations}

Strengths of the present study includes its longitudinal design, inclusion of communitydwelling cases, as well as detailed neuropsychological testing at baseline and followup. However, some limitations should also be considered. Consistent with recent core clinical criteria for MCI [42], the identification of deteriorating controls (dCON) was based on the objective decline in cognitive functions measured using serial, comprehensive neuropsychological assessments. However, in the absence of longer follow-up the cognitive fate of these cases remains uncertain so that they cannot be $a$ priori considered as incipient $A D$ cases. No cerebrospinal fluid (CSF) measures of tau and Aß protein were available in this work so that the real extent of AD pathology remains unknown. Future studies exploring the tau and amyloid status based on positron emission tomography (PET) in our communitybased cohort are warranted to examine the relationship between AD molecular events and MRI atrophy in limbic areas in dCON. Moreover, the present experiment does not include an at rest fMRI condition. We cannot thus exclude that observed group differences may partly reflect intrinsic network alterations in $\mathrm{dCON}$ and $\mathrm{MCI}$ that are independent of output task. In fact, a recent meta-analysis showed regional resting-state differences in amnestic MCI cases relative to controls, including the posterior cingulate cortex, right angular gyrus, right parahippocampal gyrus, left fusiform gyrus, left supramarginal gyrus and bilateral middle temporal gyri [43]. However, this bias is controlled by our experimental design that was based on the fMRI contrasts between 2 back and 0 back.

\section{Conclusion}

The present study aims at investigating differences in working memory performance both at the behavioral and at the brain functional level as markers of early cognitive decline. Our results show that a disruption in the FPN activation and an increase of DMN activity (decreased deactivation) in cingulate cortices and insula are increasingly distinguishable between sCON, dCON and MCI cases. The identification of fMRI alterations during the successful performance of $n$-back task in dCON cases complete our previous investigations 
in this field that revealed both functional (arterial spin labeling decrease, altered alpha and beta brain oscillations; [16, 44] and structural changes (atrophy of the limbic system;[15] preceding the cognitive decline in this group. Taken together, these observations allows for identifying brain imaging biomarkers that could predict subsequent cognitive deterioration at the pre-MCI state.

\section{Abbreviations}

ACC (Anterior Cingulate Cortex); AD (Alzheimer Disease); ANOVA (Analysis of Variance); dCON (Deteriorated Controls); DMN (Default Mode Network); FEAT (FMRI Expert Analysis Tool); FLAME (FMRIB's Local Analysis of Mixed Effects); fMRI (Functional magnetic resonance imaging); FPN (Fronto-pariteal Network); MCI (Middle Cognitive Impairment); MMSE (Mini-mental State Examination); PCC (Posterior Cingulate Cortex); PET (Positron emission tomography); sCON (Stable Controls).

\section{Funding}

This work is supported in part by Swiss National Foundation Grant SNF 3200B01161193 and SPUM 33CM30-124111 and an unrestricted grant from the Association pour la Recherche Alzheimer Suisse.

\section{Acknowledgments}

We want to thank all the subjects that participated in this study. We want to thank Biomedical Research and Training for the technical support in this study.

\section{Disclosure Statement}

The authors declare no conflicts of interest.

\section{References}

1 Jack CR, Knopman DS, Jagust WJ, Shaw LM, Aisen PS, Weiner MW, Petersen RC, Trojanowski JQ: Hypothetical model of dynamic biomarkers of the Alzheimer's pathological cascade. Lancet Neurol 2010;9:119.

2 Alhola P, Polo-Kantola P: Sleep deprivation: Impact on cognitive performance. Neuropsychiatr Dis Treat 2007;3:553-567.

3 Glisky EL: Changes in Cognitive Function in Human Aging; in Riddle DR (ed): Brain Aging: Models, Methods, and Mechanisms. Boca Raton (FL), CRC Press/Taylor \& Francis, 2007, [cited 2017 Mar 16]. Available from: http://www.ncbi.nlm.nih.gov/books/NBK3885/.

4 Baddeley A: Working memory. Science 1992;255:556-559.

5 Raichle ME: The brain's default mode network. Annu Rev Neurosci 2015;38:433-447.

6 Bartova L, Meyer BM, Diers K, Rabl U, Scharinger C, Popovic A, Pail G, Kalcher K, Boubela RN, Huemer J, Mandorfer D, Windischberger C, Sitte HH, Kasper S, Praschak-Rieder N, Moser E, Brocke B, Pezawas L: Reduced default mode network suppression during a working memory task in remitted major depression. J Psychiatr Res 2015;64:9-18.

7 Kim SG, Uğurbil K: Comparison of blood oxygenation and cerebral blood flow effects in fMRI: estimation of relative oxygen consumption change. Magn Reson Med 1997;38:59-65. 
Neurosignals 2017;25:127-138

\begin{tabular}{|l|l|l|}
\hline DOI: 10.1159/000486152 & (c) 2017 The Author(s). Published by S. Karger AG, Basel \\
\hline
\end{tabular}

Published online: January 15, 2017

www.karger.com/nsg

Zanchi et al:: Working Memory Performance and Changes in Neural Activation in Cognitively Preserved Elders

8 Koshino H, Minamoto T, Yaoi K, Osaka M, Osaka N: Coactivation of the Default Mode Network regions and Working Memory Network regions during task preparation. Sci Rep 2014;4:5954.

9 Cabeza R, Nyberg L: Imaging cognition II: An empirical review of 275 PET and fMRI studies. J Cogn Neurosci 2000;12:1-47.

10 Corbetta M, Shulman GL: Control of goal-directed and stimulus-driven attention in the brain. Nat Rev Neurosci 2002;3:201-215.

11 Mammarella N, Fairfield B: Emotional Working Memory and Alzheimer's Disease. Int J Alzheimers Dis 2014;2014. DOI: 10.1155/2014/207698

12 Yetkin FZ, Rosenberg RN, Weiner MF, Purdy PD, Cullum CM: FMRI of working memory in patients with mild cognitive impairment and probable Alzheimer's disease. Eur Radiol 2006;16:193-206.

13 Niu H-J, Li X, Chen Y-J, Ma C, Zhang J-Y, Zhang Z-J: Reduced frontal activation during a working memory task in mild cognitive impairment: a non-invasive near-infrared spectroscopy study. CNS Neurosci Ther 2013;19:125-131.

14 Lazarczyk MJ, Hof PR, Bouras C, Giannakopoulos P: Preclinical Alzheimer disease: identification of cases at risk among cognitively intact older individuals. BMC Med 2012;10:127.

15 Zanchi D, Giannakopoulos P, Borgwardt S, Rodriguez C, Haller S: Hippocampal and Amygdala Gray Matter Loss in Elderly Controls with Subtle Cognitive Decline. Front Aging Neurosci 2017;9. DOI: 10.3389/ fnagi.2017.00050

16 Xekardaki A, Rodriguez C, Montandon M-L, Toma S, Tombeur E, Herrmann FR, Zekry D, Lovblad KO, Barkhof F, Giannakopoulos P, Haller S: Arterial spin labeling may contribute to the prediction of cognitive deterioration in healthy elderly individuals. Radiology 2015;274:490-499.

17 Pronk M, Deeg DJH, Kramer SE: Hearing status in older persons: a significant determinant of depression and loneliness? Results from the longitudinal aging study amsterdam. Am J Audiol 2013;22:316-320.

18 Folstein MF, Folstein SE, McHugh PR: “Mini-mental state”. A practical method for grading the cognitive state of patients for the clinician. J Psychiatr Res 1975;12:189-198.

19 Zigmond AS, Snaith RP: The hospital anxiety and depression scale. Acta Psychiatr Scand 1983;67:361-370.

20 Barberger-Gateau P, Commenges D, Gagnon M, Letenneur L, Sauvel C, Dartigues JF: Instrumental activities of daily living as a screening tool for cognitive impairment and dementia in elderly community dwellers. J Am Geriatr Soc 1992;40:1129-1134.

21 Reitan RM: Validity Of The Trail Making Test As An Indicator Of Organic Brain Damage. Percept Mot Skills 1958;8:271.

22 Wechsler D: Wechsler Memory Scale. Psychological Corporation, 1997.

23 Milner B: Interhemispheric differences in the localization of psychological processes in man. Br Med Bull 1971;27:272-277.

24 Buschke H, Sliwinski MJ, Kuslansky G, Lipton RB: Diagnosis of early dementia by the Double Memory Test: encoding specificity improves diagnostic sensitivity and specificity. Neurology 1997;48:989-997.

25 Kaplan E, Goodglass H, Weintraub S, Goodglass H: Boston naming test. Philadelphia, Lea \& Febiger, 1983.

26 Schnider A, Hanlon RE, Alexander DN, Benson DF: Ideomotor apraxia: behavioral dimensions and neuroanatomical basis. Brain Lang 1997;58:125-136.

27 Poeck K: Clues To The Nature of Disruptions To Limb Praxis. Adv Psychol 1985;23:99-109.

28 Welsh KA, Butters N, Mohs RC, Beekly D, Edland S, Fillenbaum G, Heyman A: The Consortium to Establish a Registry for Alzheimer's Disease (CERAD). Part V. A normative study of the neuropsychological battery. Neurology 1994;44:609-614.

29 Hughes CP, Berg L, Danziger WL, Coben LA, Martin RL: A new clinical scale for the staging of dementia. Br J Psychiatry J Ment Sci 1982;140:566-572.

30 Petersen RC, Doody R, Kurz A, Mohs RC, Morris JC, Rabins PV, Ritchie K, Rossor M, Thal L, Winblad B: Current concepts in mild cognitive impairment. Arch Neurol 2001;58:1985-1992.

31 Haller S, Borgwardt SJ, Schindler C, Aston J, Radue EW, Riecher-Rössler A: Can cortical thickness asymmetry analysis contribute to detection of at-risk mental state and first-episode psychosis? A pilot study. Radiology 2009;250:212-221.

32 Sinanaj I, Montandon M-L, Rodriguez C, Herrmann F, Santini F, Haller S, Giannakopoulos P: Neural underpinnings of background acoustic noise in normal aging and mild cognitive impairment. Neuroscience 2015;310:410-421. 
33 Belleville S, Chertkow H, Gauthier S: Working memory and control of attention in persons with Alzheimer's disease and mild cognitive impairment. Neuropsychology 2007;21:458-469.

34 Kirova A-M, Bays RB, Lagalwar S: Working Memory and Executive Function Decline across Normal Aging, Mild Cognitive Impairment, and Alzheimer's Disease. BioMed Res Int 2015;2015. DOI: $10.1155 / 2015 / 748212$

35 Darki F, Klingberg T: The Role of Fronto-Parietal and Fronto-Striatal Networks in the Development of Working Memory: A Longitudinal Study. Cereb Cortex 2015;25:1587-1595.

36 Harding IH, Yücel M, Harrison BJ, Pantelis C, Breakspear M: Effective connectivity within the frontoparietal control network differentiates cognitive control and working memory. Neurolmage 2015;106:144-153.

37 Yeung MK, Sze SL, Woo J, Kwok T, Shum DHK, Yu R, Chan AS: Reduced Frontal Activations at High Working Memory Load in Mild Cognitive Impairment: Near-Infrared Spectroscopy. Dement Geriatr Cogn Disord 2016;42:278-296.

38 Vermeij A, Kessels RPC, Heskamp L, Simons EMF, Dautzenberg PLJ, Claassen JAHR: Prefrontal activation may predict working-memory training gain in normal aging and mild cognitive impairment. Brain Imaging Behav 2017;11:141-154.

39 Liang X, Zou Q He Y, Yang Y: Topologically Reorganized Connectivity Architecture of Default-Mode, Executive-Control, and Salience Networks across Working Memory Task Loads. Cereb Cortex N Y N 1991 2016;26:1501-1511.

40 Vatansever D, Manktelow AE, Sahakian BJ, Menon DK, Stamatakis EA: Angular default mode network connectivity across working memory load. Hum Brain Mapp 2017;38:41-52.

41 Čeko M, Gracely JL, Fitzcharles M-A, Seminowicz DA, Schweinhardt P, Bushnell MC: Is a Responsive Default Mode Network Required for Successful Working Memory Task Performance? J Neurosci Off J Soc Neurosci 2015;35:11595-11605.

42 Albert MS, DeKosky ST, Dickson D, Dubois B, Feldman HH, Fox NC, Gamst A, Holtzman DM, Jagust WJ, Petersen RC, Snyder PJ, Carrillo MC, Thies B, Phelps CH: The diagnosis of mild cognitive impairment due to Alzheimer's disease: recommendations from the National Institute on Aging-Alzheimer's Association workgroups on diagnostic guidelines for Alzheimer's disease. Alzheimers Dement J Alzheimers Assoc 2011;7:270-279.

43 Lau WKW, Leung M-K, Lee TMC, Law ACK: Resting-state abnormalities in amnestic mild cognitive impairment: a meta-analysis. Transl Psychiatry 2016;6:e790.

44 Deiber M-P, Meziane HB, Hasler R, Rodriguez C, Toma S, Ackermann M, Herrmann F, Giannakopoulos P: Attention and Working Memory-Related EEG Markers of Subtle Cognitive Deterioration in Healthy Elderly Individuals. J Alzheimers Dis JAD 2015;47:335-349. 